\title{
Relação entre habilidades espaciais e desempenho no ensino médio
}

La relación entre las habilidades espaciales y lo rendimento en la secundaria Relationship between spatial abilities and performance in high school

\author{
Diego Vinícius da Silva \\ Universidade São Francisco, Itatiba, SP, Brasil \\ Maria Cristina Rodrigues Azevedo Joly \\ Universidade São Francisco, Itatiba, SP, Brasil \\ Gerardo Prieto \\ Universidad de Salamanca, Salamanca, Espanha
}

\section{Resumo}

A visualização espacial é definida como a capacidade para gerar uma imagem mental, avaliar as transformações e armazenar as modificações produzidas. Pesquisas revelam que a visualização espacial, enquanto fator da habilidade espacial está diretamente relacionada com o bom desempenho escolar, principalmente, em Matemática, Física e Geografia, as quais exigem raciocínio para solucionar problemas. Assim, o objetivo do presente estudo foi identificar as correlações entre o desempenho de estudantes nas disciplinas de matemática, física e geografia com as habilidades de visualização e raciocínio espacial, avaliadas por meio de dois testes psicológicos. Participaram 116 estudantes do Ensino Médio, sendo 58,62\% do gênero feminino com as idades variaram entre 14 e 18 anos $(M=16,09 ; D P=0,98)$. Constatouse correlação positiva estatisticamente significativa entre a visualização espacial e as disciplinas de matemática e geografia, entre a habilidade de visualização espacial e raciocínio espacial, e entre raciocínio espacial e matemática, física e geografia.

Palavras-chave: Avaliação Psicológica, Visualização Espacial, Raciocínio Espacial, Desempenho Escolar.

\section{Resumen}

La visualización espacial se define como la capacidad de generar una imagen mental, las transformaciones y guardar los cambios realizados. La investigación demuestra quela visualización espacial, un factor de capacidad espacial está directamente relacionada 
con el rendimiento académico, especialmente en matemáticas, física y geografía, que requieren razonamiento para resolver problemas. El objetivo de este estudio fue identificar las correlaciones entre el rendimiento de los estudiantes en matemáticas, física y geografía con las habilidades de la visualización y el razonamiento espacial, evaluada mediante dos pruebas psicológicas. En este estudio participaron 116 estudiantes de secundaria (58,62\% mujeres), con edades que oscilaron entre 14 y 18 años $(\mathrm{M}=16.09, \mathrm{SD}=0,98)$. Se encontró una correlación positiva estadísticamente significativa entre la visualización espacial, las Matemáticas y la Geografía; entre la capacidad de visualización espacial y el razonamiento espacial, y entre el razonamiento espacial las Matemáticas, la Física y la Geografía.

Palabras clave: Evaluación Psicológica, Visualización Espacial; Razonamiento Espacial, El Rendimiento Escolar.

\begin{abstract}
The visualization space is defined as the ability to generate a mental image, the transformations and store the changes made. Research shows that the spatial visualization, a factor of spatial ability is directly related to academic achievement, especially in math, physics and geography, which require reasoning to solve problems. The objective of this study was to identify correlations between the performance of students in math, physics and geography with the skills of visualization and spatial reasoning, assessed by means of two psychological tests. 116 students participated in high school, and 58.62\% females with ages ranging between 14 and 18 years $(M=16.09 ; S D=0.98)$. It was found statistically significant positive correlation between spatial visualization and math and geography, between the ability of spatial visualization and spatial reasoning, and between spatial reasoning and mathematics, physics and geography.
\end{abstract}

Keywords: Psychological Assessment, Spatial Visualization, Spatial Reasoning, School Performance.

A habilidade espacial foi o tema central desse estudo, que visou avaliar dois componentes associados na literatura como representativos dessa habilidade, a saber, a visualização espacial e o raciocínio espacial. Além disso, observou-se por meio das notas escolares o desempenho de estudantes nas disciplinas de Geografia, Matemática e Física. Nesse sentido, essa avaliação foi realizada procurando responder a seguinte questão: As habilidades espaciais, visualização e 
raciocínio espacial, estão relacionados ao bom desempenho de estudantes no Ensino Médio? Considerando essa pergunta, a revisão teórica realizada nesse estudo procura delimitar esses construtos apontando suas acepções enquanto aspectos relacionados à inteligência geral, bem como, descreve-se pesquisas científicas que procuraram estudar sobre habilidades espaciais e desempenho escolar.

A inteligência é um dos construtos psicológicos mais estudados e avaliados pelos pesquisadores da área de Psicologia, mas há ainda controvérsias quanto a sua definição e formas de avaliação (Almeida, Guisande, Primi \& Ferreira, 2008). Há autores que a compreendem na sua generalidade, ou seja, uma capacidade geral de pensamento que se exprime num fator geral $(g)$. Outros estão atentos à sua natureza multifacetada, sugerindo que as diferenças individuais no desempenho não podem ser atribuídas aos níveis de capacidade num fator geral, mas também a um conjunto de habilidades básicas (Flanagan, Ortiz, Alfonso \& Mascolo, 2002; Primi, 2003; Almeida \& cols, 2008).

Segundo Almeida e cols. (2008) a Teoria das Capacidades Cognitivas de Cattell-Horn-Carroll (CHC), enquanto teoria fatorial de inteligência agrega a possibilidade de avaliar tanto a habilidade considerada como inerente ao indivíduo, relacionada à capacidade de solucionar problemas (raciocínio e inteligência fluida) quanto à influenciada pela aprendizagem e cultura (inteligência cristalizada). Essa teoria especifica dez fatores de domínio das capacidades cognitivas, a saber, a Inteligência fluida (Gf); Conhecimento quantitativo $(G q)$; Inteligência cristalizada $(G c)$; Memória a curto prazo (Gsm); Processamento visual $(G v)$; Processamento auditivo $(G a)$; Armazenamento e recuperação associativa a longo prazo (Glr); Velocidade de processamento cognitivo $(G s)$; Velocidade de decisão $(G t)$; e Leitura-escrita $(G r w)$. Dentre essas capacidades cognitivas destaca-se a de processamento visual $(G v)$, que envolve a habilidade para processar estímulos visuais, incluindo gerar, perceber, armazenar, analisar, manipular e transformar imagens visuais.

Estudos empíricos e teóricos envolvendo a representação mental da informação espacial têm sido desenvolvidos desde a década de 1970 pela Psicologia Cognitiva (De Vega \& Marschark, 1996). Porém, apesar da importância da aptidão espacial observada nas teorias sobre a inteligência, os testes de orientação espacial não têm sido utilizados para a seleção de pessoal e para o diagnóstico educativo (Prieto \& Velasco, 2006; Webb, Lubinski \& Benbow, 2007). Os sistemas de percepção visual 
captam as informações do ambiente por meio das propriedades visuais dos objetos, relações espaciais entre eles e suas funções dentro do ambiente. A informação visoespacial, registrada na memória, permite a re-experimentação perceptual anterior, assim pode-se combinar elementos visoespaciais de novas maneiras, realizar transformações sobre elas e engajá-las no raciocínio e resolução de problemas que envolvem informações espaciais (De Vega \& Marschark, 1996).

Lohman (1993) considera a relação espacial, a orientação espacial e a visualização espacial como os três grandes fatores da habilidade espacial, mas a visualização espacial é considerada um dos fatores mais representativos da habilidade espacial (Lohman, 2000; Prieto \& Velasco, 2002; Prieto, 2008). Essa habilidade é definida como a capacidade para manipular mentalmente figuras tridimensionais complexas, e, a habilidade para gerar uma imagem mental, avaliar as transformações e armazenar as modificações produzidas (Lohman, 1993; Prieto \& Velasco, 2002). Em relação à sua avaliação, os testes mais utilizados são compostos por tarefas de quebra-cabeça, cubos, desenvolvimento de superfícies, dobradura de papel, transformações em duas dimensões (2D) e três dimensões (3D) (Olkun, 2003; Prieto, 2008).

Em pesquisa realizada com estudantes alemães, Rammstedt e Rammsayer (2000) avaliaram os escores de inteligência e verificaram sua relação com o desempenho em matemática de 105 estudantes universitários de psicologia, sendo 54 do gênero masculino. Encontraram correlações significativas entre inteligência matemática e espacial $(r=0,27 ; p<0,05)$ e raciocínio $(r=0,56$; $p<0,001)$, e, entre inteligência espacial e raciocínio $(r=0,38 ; p<0,01)$ para o gênero masculino. Para as mulheres houve também correlações positivas entre matemática e habilidade espacial $(r=0,51$; $p<0,001)$ e raciocínio $(r=0,53 ; p<0,001)$, e entre habilidades espacial e raciocínio $(r=0,45 ; p<0,01)$.

Com o objetivo de investigar a utilização da imagem visual e sua relação com a habilidade de visualização espacial, Garderen (2006) realizou um estudo com 66 alunos de sexta série em uma escola da Flórida. Os alunos foram agrupados de acordo com sua habilidade de resolver problemas (dificuldades de aprendizagem, com desempenho médio, e os talentosos). Os resultados, por meio da ANOVA, revelaram existir diferenças em função do desempenho em exercícios matemáticos $(F(2,63)=42.58, \quad p<0,001)$, a prova de Tukey indicou que os alunos mais talentosos apresentaram uma maior habilidade de visualização espacial do que os alunos com dificuldades e com 
desempenho médio. $\mathrm{O}$ desempenho na resolução de problemas matemáticos se correlacionou com o uso de imagens visuais $(r=0,38 ; \quad p<0,01)$ e com a visualização espacial $(r=0,68 ; p<0,01)$.

Webb, Lubinski e Benbow (2007) realizaram uma pesquisa para determinar se a habilidade espacial poderia ser utilizada como critério de identificação de estudantes promissores na área de ciência. Para tanto, selecionaram estudantes em programas de talentos/superdotados. Os dados dos participantes no Scholastic Aptitude Test (SAT) foram analisados longitudinalmente, no qual a habilidade espacial revelou correlação positiva com matemática $\quad(r=0,24 ; \quad p<0,0001)$. Os resultados indicaram que a capacidade espacial pode ser acrescentada aos procedimentos de identificação de estudantes intelectualmente talentosos em Matemática.

O raciocínio, por sua vez, está associado a outros construtos (e.g. inteligência, resolução de problemas e compreensão/pensamento), e foi definido como o exercício cognitivo que as pessoas utilizam para solucionar problemas diferenciados quanto ao seu conteúdo (numérico, verbal, espacial, abstrato e mecânico). O raciocínio é responsável pelos componentes de tratamento e relacionamento da informação, sendo um dos elementos mais importantes na resolução de problemas, principalmente quando as tarefas a serem resolvidas exigem descobertas de relações, comparação de elementos e a escolha de uma entre várias alternativas propostas como resposta (Almeida, 1988).

Primi e Almeida (2000) realizaram um estudo que teve por objetivo investigar a validade e precisão da Bateria de Provas de Raciocínio (BPR-5). A bateria foi aplicada em 1243 alunos, sendo 771 brasileiros e 472 portugueses, da sexta série do Ensino Fundamental até a terceira série do Ensino Médio. Nos alunos brasileiros a prova de RE obteve correlações significativas com a disciplina de Matemática $(r=0,26 ; p<0,01)$ e Física $(r=0,36 ; p<0,05)$. Já na amostra portuguesa a prova de RE também se correlacionou com a disciplina de Matemática $(r=0,27$; $p<0,05)$, observaram-se, portanto, que há indícios de que quando o conteúdo dos subtestes e da disciplina escolar se aproximam, as correlações tendem a serem maiores.

De modo geral, as pesquisas na área de avaliação psicológica (Primi \& Almeida, 2000; Rammstedt \& Rammsayer, 2000; Garderen, 2006; Webb, Lubinski e Benbow, 2007) têm indicado que a habilidade espacial está diretamente relacionada com o bom desempenho escolar, principalmente, nas disciplinas correlatas (e.g. Matemática, Física e 
Geografia). Além disso, pesquisas relatadas por Lohman (1993) e por Prieto (2008) indicam que os testes de habilidades espaciais são conhecidos como medidas de habilidades práticas e mecânicas, e revelam-se úteis para predizer o sucesso em profissões técnicas ligadas às engenharias (e.g. mecânica, arquitetura, desenho técnico), à aeronáutica (e.g. pilotos, controladores de vôo) e à saúde (e.g. cirurgia, odontologia).

No Brasil, o processo de educacional no Ensino Médio se divide em três áreas de conhecimento descritas nos Parâmetros Curriculares Nacionais do Ensino Médio (PCNEM) Essas áreas são Linguagens, códigos e suas tecnologias; Ciências da Natureza, Matemática e suas tecnologias; e Ciências Humanas e suas tecnologias (Ministério da Educação MEC, 2006).

$\mathrm{Na}$ área de Linguagens, códigos e suas tecnologias estão descritos os conteúdos e a organização curricular das disciplinas de Língua Portuguesa, Literatura, Línguas Estrangeiras, Artes e Educação Física. Na área de Ciências da Natureza, Matemática e suas tecnologias apresentam-se a composição dos currículos das disciplinas de Biologia, Física, Matemática e Química. Na terceira e última área, Ciências Humanas e suas tecnologias incluem-se as disciplinas de Filosofia, Geografia, História e Sociologia.
(MEC, 2006). Destaca-se das duas últimas áreas as disciplinas de Matemática, Física e Geografia.

$\mathrm{Na}$ disciplina de Matemática espera-se que os estudantes saibam usá-la para resolver problemas práticos do cotidiano, verifica-se que dentre os seus conteúdos há o estudo da Geometria, por meio dele visa-se possibilitar que o aluno seja capaz de orientar-se no espaço geográfico, ler mapas, estimar e comparar distâncias. No ensino de Física devem-se organizar modelos da realidade para entendê-la e obter meios para encarar um problema, estimula-se o estudante para que desenvolva uma competência investigativa, ou seja, devem-se incitar as perguntas de modo que o conhecimento dos fenômenos da realidade passe pela abstração. A disciplina de Geografia deve preparar o aluno para localizar, compreender e atuar no mundo, reconhecer as dinâmicas existentes no espaço geográfico, pensar e atuar em sua realidade tendo em vista a sua transformação, engloba também, a dinâmica espacial, que produz, reproduz e transforma o espaço geográfico (MEC, 2006).

Considerando que a habilidade espacial se refere a um dos fatores da inteligência, mas sua avaliação é pouco realizada por psicólogos na área de avaliação psicológica (Prieto \& Velasco, 2006; Webb, Lubinski \& Benbow, 2007), 
esse estudo visa avaliar essa habilidade em estudantes. Além disso, constata-se que se a habilidade espacial deve ser desenvolvida na educação básica (MEC, 2006), pois é utilizada para a resolução de problemas práticos. Isso posto, esta pesquisa tem o objetivo geral de identificar as correlações existentes entre o desempenho de estudantes de Ensino Médio nas disciplinas de Matemática, Física e Geografia com as habilidades de visualização e raciocínio espacial. Além disso, entre os objetivos específicos estão verificar se essas habilidades espaciais se correlacionam e se diferem em função do gênero, idade e série escolar.

\section{Método}

\section{Participantes}

Participaram dessa pesquisa 116 estudantes matriculados no Ensino Médio, sendo que 58,62\% eram do gênero feminino. As idades se distribuíram entre 14 e 18 anos, sendo que a idade média foi de 16,09 $(D P=0,98)$. Em relação às séries, verificou-se que $39,5 \%$ estavam no $1^{\circ}$ ano, $34,5 \%$ no $2^{\circ}$ ano e $26,1 \%$ no $3^{\circ}$ ano do Ensino Médio de uma escola pública localizada no interior do estado de São Paulo.

\section{Instrumentos}

Para avaliar o desempenho nas disciplinas solicitou-se com a coordenação da escola o acesso às notas finais dos estudantes, que variavam de 0 a 10 pontos. Para mensurar os construtos psicológicos de interesse no estudo utilizaram-se os dois instrumentos descritos abaixo.

Teste Informatizado de Visualização Espacial (TVZ) (Prieto, 2004)

Trata-se de um instrumento informatizado de medida de visualização espacial, no qual os itens foram construídos a partir de indicadores cognitivos. A prova é constituída por 20 itens, no qual a tarefa consiste em um cubo que tem todas as faces identificadas com letras. À direita do cubo, aparece seu desdobramento no plano com uma de suas faces identificada e outra marcada com uma interrogação (?). Pedese ao sujeito que identifique a letra da face marcada com a interrogação e sua aparência. $\mathrm{O}$ examinado deve escolher a resposta correta entre as 09 opções fornecidas. A Figura 1 (em anexo) exemplifica essa definição.

Bateria de Provas de Raciocínio (BPR-5) (Almeida \& Primi, 2000)

A BPR-5 é constituída por duas formas (A e B), com cinco subtestes cada e com o mesmo número de itens. A Forma A aplica-se aos estudantes da sexta à oitava 
série do Ensino Fundamental e a Forma B aos alunos da primeira à terceira série do Ensino Médio. Nesse estudo utilizou-se a Forma B da seguinte prova:

\section{Prova de Raciocínio Espacial (RE):} composta de 20 itens (12 itens são comuns às Formas A e B) nos quais existem séries de cubos tridimensionais em diferentes posições que indicam movimento. Os movimentos podem ser constantes, por exemplo, sempre para a direita, ou alternados, por exemplo, para esquerda e para cima. Por meio da análise das diferentes faces pode-se descobrir o cubo que se seguiria se o movimento descoberto fosse aplicado ao último cubo da série. $\mathrm{Na}$ Figura 2 (em anexo), apresenta-se um exemplo de um item dessa prova.

\section{Procedimento}

Após a autorização da escola, pediu-se também, a autorização dos pais, por meio do Termo de Consentimento Livre e Esclarecido. Os testes foram aplicados em três séries diferentes, em cada turma foram necessários dois dias, previamente agendados com a direção das escolas, para a aplicação dos dois instrumentos utilizados nesse estudo. $\mathrm{Na}$ primeira sessão aplicou-se a provas da RE da BPR-5, com duração de 18 minutos por série. E na segunda sessão aplicou-se na sala de informática o Teste Informatizado de Visualização Espacial. As notas foram solicitadas na secretaria da escola.

\section{Resultados}

O desempenho dos estudantes em visualização e raciocínio espacial foi medido por meio de dois instrumentos específicos, já o desempenho nas disciplinas considerou-se as notas finais dos estudantes. Na Tabela 1 (em anexo) pode-se observar o desempenho médio dos estudantes nos dois testes e nas disciplinas de Matemática, Física e Geografia.

Considerando o desempenho médio dos estudantes verificou-se que houve baixo desempenho dos estudantes no teste de visualização espacial, pois a média foi inferior a $25 \%$ de aproveitamento considerando a nota máxima de 20 pontos.

A Figura 3 (em anexo) apresenta a distribuição de frequência dos estudantes por pontuação nesse teste.

A maior parte dos estudantes $(n=20)$ acertou apenas dois itens do TVZ, houve poucos estudantes que acertaram mais que sete questões. Destaca-se que seis estudantes não acertaram nenhum item do teste de visualização espacial.

Observa-se que no teste de raciocínio espacial a média foi inferior a $50 \%$ de aproveitamento considerando que a nota máxima também poderia ser de 20 pontos, mas dois estudantes acertaram 19 
das 20 questões. A Figura 4 (em anexo) apresenta a distribuição de frequência dos estudantes por pontuação nesse teste.

A maior parte dos estudantes $(n=14)$ acertou doze das vinte questões do teste de raciocínio espacial. Apenas um estudante não acertou nenhuma questão da prova.

Já em relação ao desempenho escolar podem-se afirmar, considerando as médias gerais, que os estudantes obtiveram um bom desempenho. A maior média foi obtida na disciplina de Matemática $(M=7,16 ; D P=1,56)$ e a menor média foi na disciplina de geografia $(M=6,40$; $D P=1,26)$.

Para verificar a correlação entre visualização e raciocínio espacial com o desempenho escolar utilizou-se o teste de correlação de Pearson. A Tabela 2 apresenta os dados dessa análise.

Tabela 2 (em anexo)

No que diz respeito à visualização espacial, constatou-se que houve correlação positiva e estatisticamente significativa com as disciplinas de matemática e geografia. Isso significa que os estudantes que apresentaram bom desempenho nessas disciplinas também apresentaram bom desempenho no teste de visualização espacial. Pode-se constatar correlações positivas estatisticamente significativas entre raciocínio espacial e matemática, física e geografia (Tabela 2).
Encontrou-se também correlação positiva e estatisticamente significativa entre a habilidade de visualização especial e o raciocínio espacial $(r=0,28 ; p=0,007)$, o que indica que os construtos visualização espacial e raciocínio espacial estão associados positivamente.

Com o objetivo de verificar se essas habilidades se diferenciavam em função do gênero dos estudantes utilizou-se o teste $t$ de Student. Nesse estudo, não se encontrou diferença estatisticamente significativa para a visualização espacial $(t(115)=-$ 0,119;p=0,903), assim como não se encontrou para o raciocínio espacial $(t(115)=-1,366 ; p=0,175)$.

Realizou-se uma Análise de Variância (ANOVA) para constatar a diferença de média em relação à idade dos participantes. Para tanto, agrupou-se os estudantes em três grupos, os estudantes com 14 e 15 anos de idade $(n=36)$ ficaram no Grupo 1, os de 16 anos $(n=43)$ no Grupo 2 e os estudantes com 17 e 18 anos de idade $(n=37)$ ficaram no Grupo 3. No teste de visualização espacial não se encontrou diferença estatisticamente significativa $(F(2,113)=1,222 ; p=0,299)$, porém houve diferença em função da idade para o raciocínio espacial $(F(2,113)=$ 4,836; $p=0,010)$. A prova post-hoc de Tukey indicou a formação de dois grupos considerando o raciocínio espacial e a idade, como pode ser observado na Tabela 
3 (em anexo).

Constata-se que os estudantes com 17 e 18 anos de idade (Grupo 3) obtiveram desempenho superior no teste de raciocínio espacial dos que os estudantes com idade inferior a 17 anos. Ressalta-se que essa diferença foi maior em relação aos estudantes com 14 e 15 anos de idade, já que o desempenho dos estudantes com 16 anos não diferenciou dos dois grupos.

Para averiguar a diferença de médias de desempenho nos testes em relação à variável série escolar também se utilizou uma ANOVA. Encontrou-se diferença estatisticamente significativa para os dois construtos como pode ser observado na Tabela 4 (em anexo).

Realizou-se o teste post-hoc de Tukey para verificar como se diferenciou o desempenho em relação às séries. A Tabela 5 apresenta os dados dessa análise.

Tabela 5 (em anexo)

O teste post-hoc de Tukey indicou a formação de dois grupos considerando a visualização espacial e a série. Os estudantes do $3^{\circ}$ ano apresentaram desempenho superior aos estudantes das outras séries, principalmente, dos estudantes do $2^{\circ}$ ano que teve $o$ menor desempenho do teste de visualização espacial. A Figura 5 (em anexo) auxilia a identificação dessas diferenças apresentando o Box-plot dos estudantes, considerando a série e a visualização espacial.

Os estudantes do $3^{\circ}$ ano apresentaram uma média superior, e, além disso, foi nessa turma que houve estudantes com pontuação maior que 10 pontos. Constatou-se, também que $50 \%$ dos estudantes do $1^{\circ}$ ano acertaram menos que três dos vinte itens do TVZ.

A prova post-hoc de Tukey também foi utilizada para verificar as diferenças de médias do raciocínio espacial por série. Essa análise indicou a formação de dois grupos considerando o raciocínio espacial e a série, como pode ser observado na Tabela 6 (em anexo).

No que tange ao raciocínio espacial, verifica-se que o desempenho acompanha a sequência das séries, ou seja, conforme aumenta as séries aumenta $\mathrm{O}$ desempenho no teste de RE. Verifica-se também, que o desempenho médio dos estudantes $2^{\circ}$, apesar ser uma média superior, não se diferenciou do desempenho dos estudantes do $1^{\circ}$ ano. A Figura 6 (em anexo) se refere ao Box-plot dos estudantes, considerando a série e raciocínio espacial.

Verifica-se que $50 \%$ dos estudantes do $3^{\circ}$ ano acertaram mais que 12 itens do teste de RE, já os estudantes do $2^{\circ}$ ano e $3^{\circ}$ ano $75 \%$ acertaram menos que 12 itens. Houve estudantes do $2^{\circ}$ ano que acertaram 18 e 19 itens, que foi a nota máxima obtida nessa amostra. 


\section{Discussão}

De modo geral, constatou-se que há relação entre visualização espacial e raciocínio espacial com o desempenho escolar. Isso significa que as habilidades requeridas no currículo do Ensino Médio (MEC, 2006) podem ser mesuradas pelos testes utilizados nesse estudo.

Referente à visualização espacial, constatou-se que houve correlação positiva estatisticamente significativa com as disciplinas de matemática e geografia. Isso indica que os estudantes que apresentaram bom desempenho nessas disciplinas também apresentaram bom desempenho no teste de visualização espacial. Tal resultado corrobora os estudos de Rammstedt e Rammsayer (2000), Garderen (2006) e Webb, Lubinski e Benbow (2007), no qual se encontraram correlações significativas entre esses construtos. Ressalta-se que o estudante no Ensino Médio deve saber usar a Matemática para resolver problemas práticos do cotidiano. Além disso, ele deve ser capaz, a partir do estudo da Geografia, de orientar-se no espaço geográfico, ler mapas, comparar distâncias, localizar, compreender e atuar no espaço geográfico (MEC, 2006), ou seja, deve desenvolver habilidades espaciais.

A visualização espacial, por sua vez, engloba a capacidade para manipular mentalmente figuras tridimensionais complexas e a habilidade para gerar uma imagem mental, avaliar as transformações e armazenar as modificações produzidas (Lohman, 1993; Prieto \& Velasco, 2002). É nesse aspecto que se relaciona a visualização espacial com o ensino de Matemática e Geografia, para o Ensino Médio, para que o estudante consiga estudar o espaço geográfico faz-se necessário que ele apresente a capacidade de manipular mentalmente suas informações geográficas.

Encontrou-se também correlação positiva estatisticamente significativa entre a habilidade de visualização especial e o raciocínio espacial. Esse dado converge com os resultados obtidos no estudo de Rammstedt e Rammsayer (2000). Portanto, na presente investigação verificou-se que as habilidades visualização e raciocínio espacial estão positivamente relacionadas, ou seja, os estudantes que apresentam alta habilidade em uma delas tendem a ter bom desempenho na outra.

Encontrou-se também, correlações positivas estatisticamente significativas entre raciocínio espacial e matemática, física e geografia. Destaca-se que a partir do ensino de matemática e física, o estudante desenvolva a capacidade de resolver problemas práticos, utilizando a abstração, e, além disso, consiga reaplicar esse conceito aprendido em outros 
problemas, criando modelos (MEC, 2006).

Esse exercício cognitivo utilizado para solucionar problemas que exigem descobertas de relações é justamente a definição de raciocínio, que é responsável também pelos componentes de tratamento e relacionamento da informação (Almeida, 1988). Em assim sendo, o raciocínio espacial é utilizado pelos estudantes investigados em relação a essas disciplinas, pois há uma relação significativa entre esses construtos (Primi \& Almeida, 2000).

\section{Considerações Finais}

As pesquisas relatadas por Lohman (1993) e por Prieto (2008) concluíram que as habilidades espaciais são habilidades preditivas para o sucesso em profissões ligadas às algumas áreas do Ensino Superior (e.g. engenharias, aeronáutica e saúde). O currículo nacional do Ensino Médio também considera importante desenvolver e avaliar diversas habilidades, no qual se destacam as habilidades espaciais (MEC, 2006). Além disso, a habilidade espacial tem sido utilizada para identificação de talentos, principalmente, se houver interesse em áreas ligado às profissões técnicas (Webb, Lubinski e Benbow, 2007), bem como, correlação positiva com bom desempenho em problemas matemáticos (Garderen, 2006) o que também foi encontrado nesse estudo.
Relativamente à avaliação do raciocínio, destaca-se principalmente a associação que vem sendo encontrada para algumas disciplinas do Ensino Básico (Almeida \& Primi, 2000) Nessa pesquisa, avaliou-se as habilidades espaciais, consideradas importantes a serem desenvolvidas no Ensino Médio (MEC, 2006) e que são também, pouco utilizadas para identificação do bom rendimento em testes de inteligência (Prieto \& Velasco, 2006; Webb \& cols., 2007). Esses dados indicam que a avaliação das habilidades espaciais pode ser útil quando se realiza, por exemplo, uma avaliação profissional, indicando que bom desempenho nesses testes pode estar relacionado a algumas profissões, revelando resultados interessantes para estudantes que se preparam para ingressar num curso superior.

Em função disso e considerando que a inteligência é formada por um conjunto de habilidades básicas (Flanagan, \& cols., 2002; Primi, 2003; Almeida \& cols, 2008), sugere-se que outros estudos busquem a avaliação da relação da visualização espacial com outros fatores da inteligência (e.g. raciocínio verbal, compreensão da leitura, memória, entre outros). Pode se verificar, também, programas objetivando desenvolver a habilidade espacial em estudantes que optarem por profissões das áreas de 
tecnologias e exatas.

\section{Referências}

Almeida, L. S. (1988). Teorias da inteligência. Porto: Edição Jornal de Psicologia.

Almeida, L. S., Guisande, M. A., Primi, R., \& Ferreira, A. (2008). Construto e medida da inteligência: construtos da abordagem fatorial. Em A. Candeias; L. Almeida, A. Roazzi, R. Primi (Orgs), Inteligência: definição $e$ medida na confluência de múltiplas concepções. São Paulo: Casa do Psicólogo.

De Vega, M. \& Marschark M. (1996) Visuospatial cognition: an historical and theoretical introduction. Em M. De Vega, M. J. Intons-Peterson, P. N. Johnson-Laird; M. Denis, \& M. Marschark (Orgs), Models of Visuospatial Cognition. New York: Oxford University Press.

Flanagan, D. P., Ortiz, S. O., Alfonso, V. C., \& Mascolo, J. T. (2002). The Achievement Test Desk Reference (ATDR): comprehensive assessment and learning disabilities. Boston: John Allyn \& Bacon.

Garderen D. V. (2006). Spatial visualization, visual imagery, and mathematical problem solving of students with varying abilities.
Journal of Learning Disabilities, 39(6) 496-506.

Lohman, D. F. (1993). Spatial Ability and G. Paper presented at the first Spearman Seminar, University of Plymouth.

Lohman, D. F. (2000). Complex information processing and intelligence. Em R. J. Sternberg (Ed.) Handbook of human intelligence. Cambridge, MA: Cambridge University Press.

Ministério da Educação (MEC), Secretaria de Educação Básica (2006). Linguagens, códigos e suas tecnologias / Secretaria de Educação Básica. Brasília, V. 1.

Ministério da Educação (MEC), Secretaria de Educação Básica (2006). Ciências da Natureza, Matemática e suas tecnologias / Secretaria de Educação Básica. Brasília, V. 2.

Ministério da Educação (MEC), Secretaria de Educação Básica (2006). Ciências Humanas e suas tecnologias / Secretaria de Educação Básica. Brasília, v. 3.

Olkun, S. (2003). Making Connections: Improving Spatial Abilities with Engineering Drawing Activities International Journal of Mathematics Teaching and Learning. [online] Disponível em <http://www.cimt.plymouth.ac.uk/jo 
urnal/sinanolkun.pdf $>$. Acessado em 20/10/2010.

Prieto, G. (2004). Teste Informatizado de Visualização Espacial. (Teste em desenvolvimento). Departamento de Psicologia, Universidad de Salamanca.

Prieto, G. (2008). Las Aptitudes Espaciales. Em A. Candeias, L. Almeida, A. Roazzi, \& R. Primi. (Orgs), Inteligência: definição $e$ medida na confluência de múltiplas concepções. São Paulo: Casa do Psicólogo.

Prieto, G., \& Velasco, A. D. (2002). Construção de um Teste de Visualização a partir da Psicologia Cognitiva. Avaliação Psicológica, 1(1), 39-47.

Prieto, G., \& Velasco, A. D. (2006). Visualização espacial, raciocínio indutivo e rendimento acadêmico em desenho técnico. Psicologia escolar e educacional, 10(1), 11-20.

Primi, R. (2003). Inteligência: avanços nos modelos teóricos e nos instrumentos de medida. Avaliação psicológica, 2(1), 67-77.

Primi, R.; Almeida, L. S. (2000). Estudo de validação da bateria de provas de raciocínio (BPR-5). Psicologia: Teoria e Pesquisa, 16 (2) 165-173.

Rammstedt, B, \& Rammsayer. T. H. (2000). Sex differences in selfestimates of different aspects of intelligence. Personality and Individual Differences, 29(2000) 869-880.

Webb, R. M., Lubinski, D., \& Benbow, C. P. (2007). Spatial ability: A neglected dimension in talent searches for intellectually precocious youth. Journal of Educational Psychology, 99(2), 397-420. 


\section{Anexos}

Tabela 1

Estatísticas descritivas dos testes TVZ e RE e das disciplinas escolares

\begin{tabular}{lcccc}
\hline Avaliações & Mínimo & Máximo & Média & $D P$ \\
\hline Visualização Espacial & 00 & 11 & 3,43 & 2,41 \\
Raciocínio Espacial & 00 & 19 & 9,34 & 4,59 \\
Matemática & 04 & 10 & 7,16 & 1,56 \\
Física & 00 & 09 & 6,58 & 1,68 \\
Geografia & 03 & 09 & 6,40 & 1,26 \\
\hline
\end{tabular}

Tabela 2

Correlação de Pearson para TVZ, RE e desempenho escolar

\begin{tabular}{llcc}
\hline Disciplinas & Índices de Correlação & Visualização Espacial & Raciocínio Espacial \\
\hline Matemática & $r$ & $0,24^{*}$ & $0,29^{*}$ \\
& $p$ & 0,019 & 0,002 \\
\hline Física & $r$ & 0,18 & $0,24^{*}$ \\
& $p$ & 0,082 & 0,010 \\
\hline Geografia & $r$ & $0,24^{*}$ & $0,23^{*}$ \\
& $p$ & 0,021 & 0,013 \\
\hline
\end{tabular}

* Correlações estatisticamente significativas.

Tabela 3

Subconjuntos formados pelo Tukey em função da idade e do desempenho no RE

\begin{tabular}{llc}
\hline & & \multicolumn{2}{c}{ Subconjunto para $\alpha=0.05$} \\
\cline { 3 - 3 } Grupos por idade & $\mathrm{N}$ & 1 \\
\hline
\end{tabular}




\begin{tabular}{llcc}
\hline Grupo 1 & 36 & 7,81 & \\
Grupo 2 & 43 & 9,16 & 9,16 \\
Grupo 3 & 37 & & 11,03 \\
\hline$p$ & & 0,378 & 0,162 \\
\hline
\end{tabular}

Tabela 4

ANOVA para TVZ e RE em função da série

\begin{tabular}{llccccc}
\hline Construtos & Série & Média & $D P$ & gl & $F$ & $p$ \\
\hline Visualização Espacial & $1^{\circ}$ Ano & 3,08 & 2,25 & 02 & 3,706 & 0,028 \\
& $2^{\circ}$ Ano & 2,97 & 2,08 & 113 & & \\
& $3^{\circ}$ Ano & 4,52 & 2,74 & 115 & & \\
\hline Raciocínio Espacial & $1^{\circ}$ Ano & 8,53 & 4,43 & 02 & 4,080 & 0,019 \\
& $2^{\circ}$ Ano & 8,83 & 4,69 & 113 & & \\
& $3^{\circ}$ Ano & 11,43 & 4,23 & 115 & & \\
\hline
\end{tabular}

Tabela 5

Subconjuntos formados pelo Tukey em função da série e do desempenho no TVZ

\begin{tabular}{lccc}
\hline \multirow{2}{*}{ Série } & & \multicolumn{2}{c}{ Subconjunto para $\alpha=0.05$} \\
\cline { 3 - 4 } & $\mathrm{N}$ & 1 & 2 \\
\hline $2^{\mathbf{o}}$ ano & 41 & 2,97 & \\
$1^{\text {o }}$ ano & 47 & 3,08 & 3,08 \\
$3^{\text {o }}$ ano & 28 & & 4,52 \\
\hline$p$ & & 0,981 & 0,051 \\
\hline
\end{tabular}


Tabela 6

Subconjuntos formados pelo Tukey em função da série e do desempenho no RE

\begin{tabular}{lccc}
\hline & & \multicolumn{2}{c}{ Subconjunto para $\alpha=0.05$} \\
\cline { 3 - 4 } Série & $\mathrm{N}$ & 1 & 2 \\
\hline $1^{\mathbf{0}}$ Ano & 47 & 8,53 & \\
$2^{\mathbf{o}}$ Ano & 41 & 8,83 & \\
$3^{\text {o Ano }}$ & 28 & & 11,43 \\
\hline$p$ & & 0,956 & 1,000 \\
\hline
\end{tabular}

Figura 5

Exemplo de um item do teste TVZ, a opção correta é a marcada com a seta

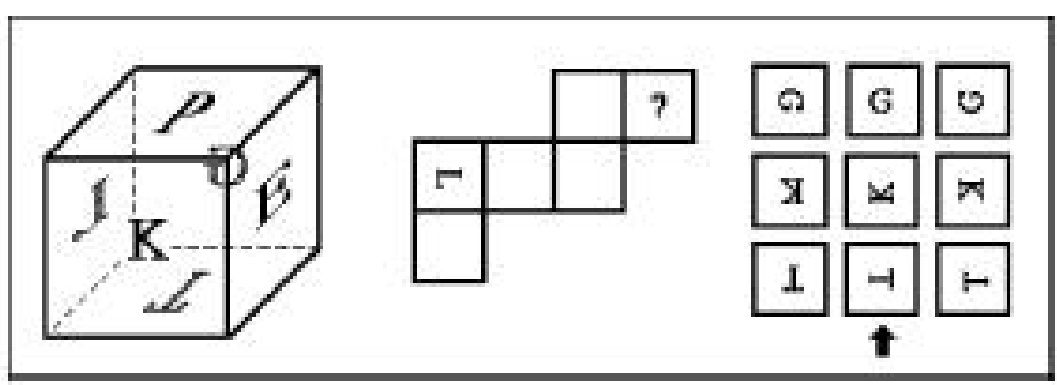

Figura 6

Exemplo de um item de raciocínio espacial

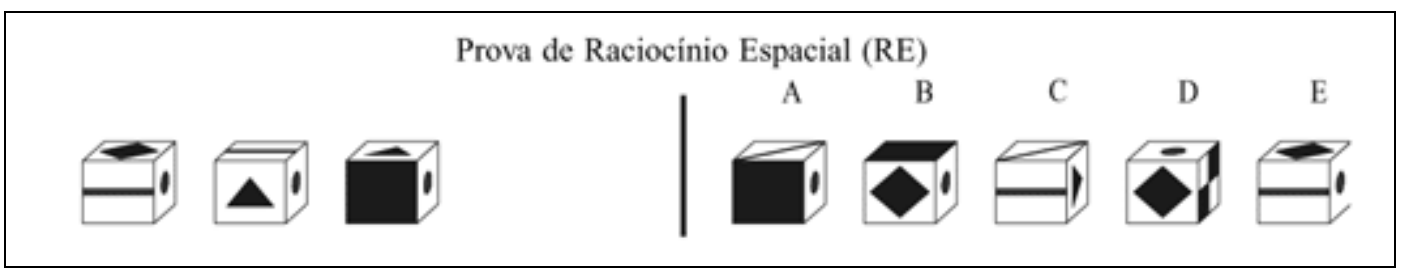


Figura 7

Distribuição dos estudantes em visualização espacial

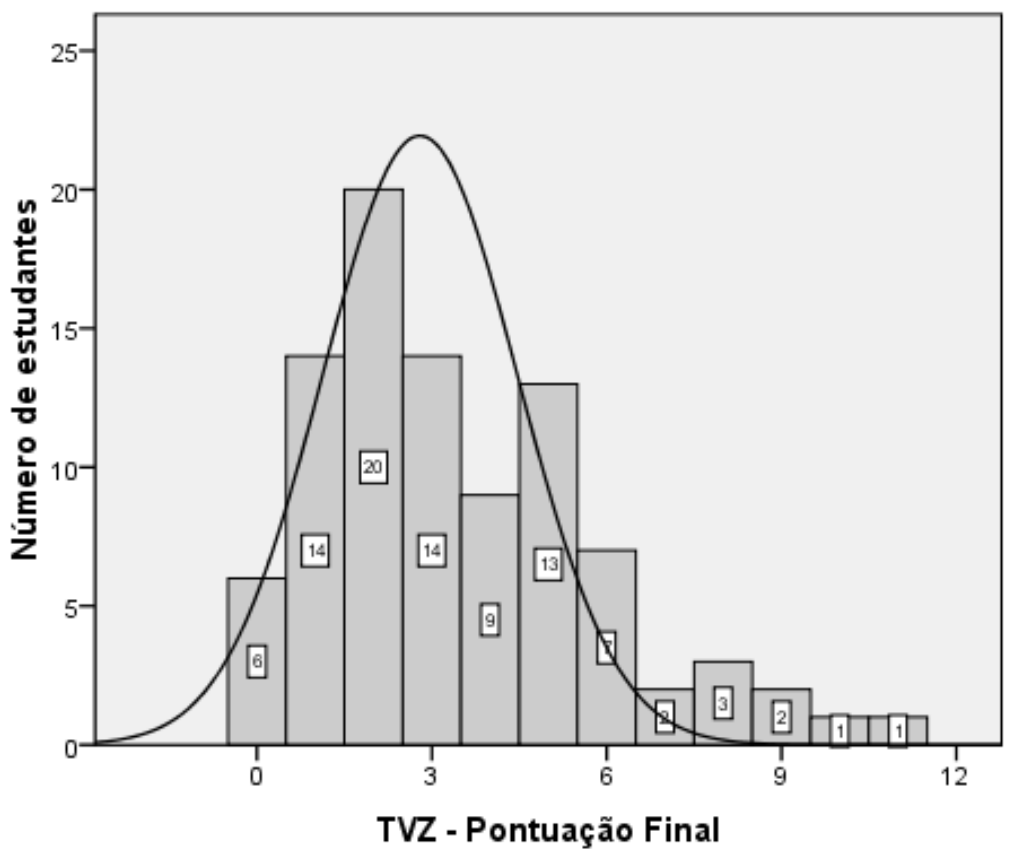

Figura 8

Distribuição dos estudantes em raciocínio espacial

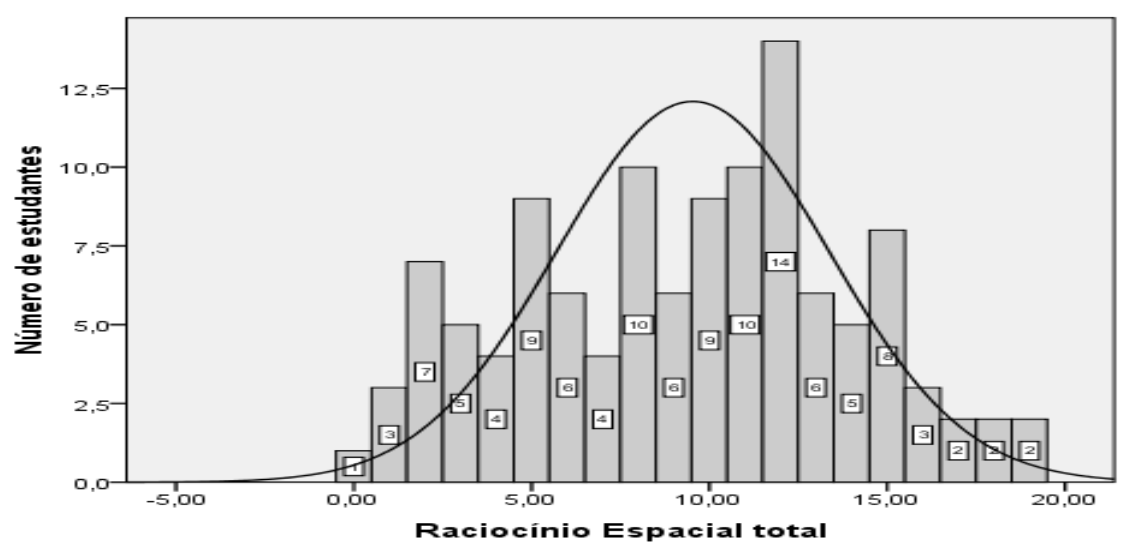


Figura 5

Box-plot dos estudantes considerando a série e a pontuação no TVZ

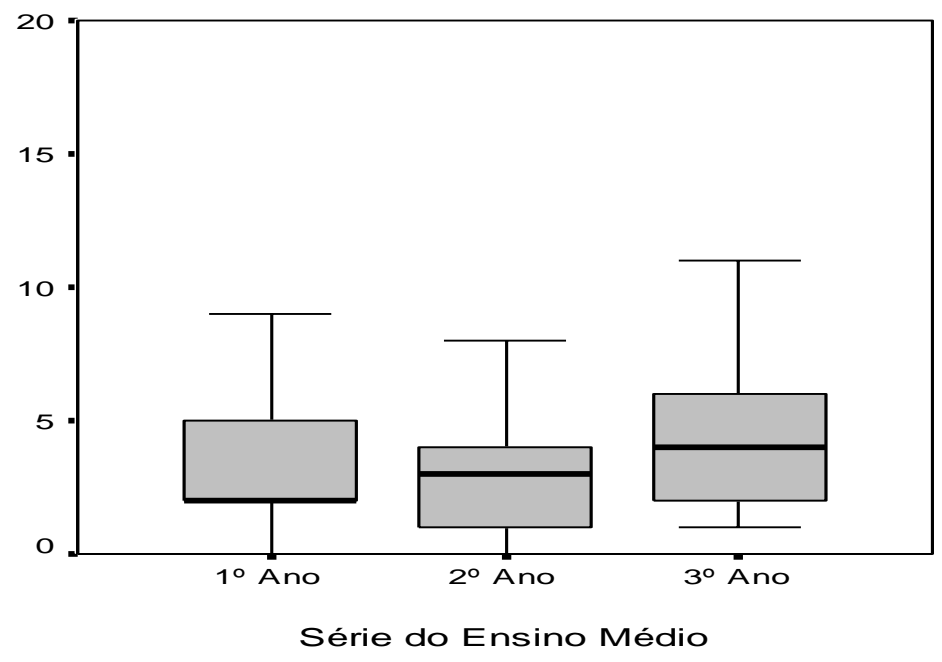

Figura 6

Box-plot dos estudantes considerando a série e raciocínio espacial

Diego Vinícius Silva. Universidade São Francisco, graduado em psicologia, doutorando em avaliação psicológica. Integrante do Núcleo de Avaliação Psicológica Informatizada.

E-mail: vinicius.diego@ymail.com

Maria Cristina Rodrigues Azevedo Joly.

Possui graduação em Psicologia (1983) e especialização em Psicologia Escolar e da Aprendizagem (1985) pela Pontifícia Universidade Católica de Campinas, mestrado (1992) e doutorado (1999) em Psicologia Escolar e do Desenvolvimento Humano pela Universidade de São Paulo e pós-doutorado em Avaliação Psicológica pela Universidade do Minho, Portugal. Atualmente é professor associado doutor da Universidade São Francisco no Programa de Pós-Graduação stricto sensu em Psicologia e no curso de graduação em Psicologia.

Gerardo Prieto. Doutor, Graduação e Pós-Graduação em Psicologia da Universidade de Salamanca (Espanha).

Endereço para correspondência: Faculdade de Ciências Sociais Aplicadas de Extrema. Estrada Municipal Pedro Rosa da Silva S/N - Vila Rica Extrema - Minas Gerais CEP: 37.640-000 - Tel: |35|3435 3988 